\section{Factors affecting glucose and insulin metabolism in young horses}

\author{
Sarah L. Ralston \\ New Brunswick, NJ
}

\section{Introduction}

High starch, high glycemic index concentrates have been implicated as a cause of increased incidence of OCD and other growth related disorders (Kronfeld et al. 1990, Ralston 1996, Ralston et al. 1996, Pagan et al. 2001, Rich and Breuer 2002). There is increasing evidence that this may be related to high insulin responses to feeding (hyperinsulinemia) and concomitant alterations in growth hormone, T-3, T-4, IGF-1, and possibly GLP-16 or leptin7 due to high glycemic indices of the feeds (Pagan et al. 2001, Ralston and Duarte 2003), or genetic defects in insulin sensitivity (Ralston 1996, Ralston et al. 1996, Cartmill et al. 2003). A single blood sample, especially if the recent dietary history of the animal and time of the last meal is not known, is basically useless as a diagnostic tool. A better understanding of both the limitations of tests of glucose metabolism and the factors affecting glucose/insulin metabolism in young horses hopefully will lead to improved nutritional recommendations that will reduce the incidence of growth disorders.

\section{Tests of Glucose/insulin metabolism}

There are various techniques to evaluate glucose/insulin metabolism in horses that measure glucose tolerance/insulin sensitivity. Traditionally "oral" (dextrose solutions delivered via nasogastric tube) or intravenous dextrose challenges that stimulated responses far above the normal physiologic range were used (Argenzio and Hintz 1972, Jacobs and Bolton 1982, Garcia and Beech 1986, Jacobs and Bolton 2002, Ralston 2002, Ralston 2004). The limitations of these challenges have been discussed previously (Jacobs and Bolton 1982, Garcia and Beech 1986, Ralston 2002, Hoffman et al. 2003a, Hoffman et al 2003b, Ralston 2004) the most significant of which are that they are not practical for use in the field, pose a non-physiologic challenge to the system and that a variety of factors, such as age, ration, physiologic status, exercise and obesity, can influence the results. Glucose "clamp" or hyperinsulinemic/hyperglycemic clamp techniques are more accurate, though still non-physiologic, assessments of glucose metabolism (Ralston 2002). Both can permit calculation of the amount of glucose disappearance per unit of insulin, if properly implemented. However both techniques are difficult and complex to administer, in that they require frequent (q 1-2 min) blood sampling for prolonged periods of time and immediate analysis of blood glucose to allow adjustment of infusion rates to maintain a steady plasma glucose concentration (Powell and Reedy 2002).
This part of the review will focus on some novel tests, which are either more physiologic and less labor intensive as research tools (Hoffman et al. 2003a, Hoffman et al. 2003b) or, on the other hand, are being used as rapid field tests to screen for abnormalities (Bergman et al. 1979).

\section{Insulin-modified Frequently Sampled Intravenous Glu- cose tolerance test (FSGIT)}

This test, often employed in human studies, provides a quantitative assessment of insulin sensitivity. In horses $0.3 \mathrm{~g} / \mathrm{kg}$ dose of glucose given intravenously has been used as the loading dose (Hoffman et al. 2003 a). Blood samples are taken at 1 to 2 min after dosing for $20 \mathrm{~min}$ but immediate analysis of blood glucose is not necessary. A relatively physiologic dose of human insulin $(30 \mathrm{mU} / \mathrm{kg} \mathrm{BW})$ is then given and blood sampling resumes every 1 to 2 min for the next ten minutes, then at frequent intervals $(5-10 \mathrm{~min})$ for the next three hours to document glucose and insulin clearance rates. Since the dose of insulin dose is $1 / 10$ the amount required to induce hypoglycemia in horses, the samples do not need to be immediately analyzed to prevent hypoglycemia, nor is there a need to rapidly adjust infusion rates. The data are evaluated using the minimal model (Powell and Reedy 2002) to calculate insulin sensitivity based on the rates of glucose appearance/disappearance before and after the insulin challenge. This technique has been used experimentally to detect subtle differences in insulin sensitivity due to fat content of the ration (Hoffman et al. 2003a), but would not be practical for use in the field.

\section{Grain test}

This is probably the least accurate and most commonly misused of the newer "field tests" being employed. Feeding 1 to 2 $\mathrm{kg}$ of a grain concentrate to a subject horse and preferably also a "control" animal can provide some information on glucose digestion, absorption and insulin responses to glucose absorption. However, the "controls" should be the same age and size with similar feeding histories and activity levels. Taking blood samples for glucose and insulin analysis before feeding and at 60,90 and 120 minutes may determine peak glucose and insulin responses, however the time the peaks occur can be influenced greatly by the rate of consumption, type of concentrate used, gastric emptying and stress (Ralston 2002, Ralston 2004). The test should be done between 0700 and $0900 \mathrm{~h}$ in the morning, since diurnal variations in cortisol will influence glucose metabolism, resulting in lower responses later in the day (Ralston 2002). Based on the authors' experience, "normal" glucose responses are usually between 100 to $130 \mathrm{mg} / \mathrm{dl}$ and insulin should not peak over $25-30 \mathrm{mlU} / \mathrm{ml}$ in horses less than 9 months of age (Ralston 2004). Insulin responses one or two times higher than normal have been correlated with the presence of radiographic evidence of hock osteochondrosis in Standardbred horses less than 9 months of age (Ralston 1996, Ralston et al. 1998). The abnormal response diminished with age, even in affected horses. If hyperinsulinemic responses are detected, it would be prudent to radiograph the hocks, stifles and fetloocks to determine the presence or absence of osteochondrotic lesions and perhaps verify the abnormality with a more accurate test. 


\section{Oral dextrose challenges}

The traditional oral dextrose challenge tests both glucose absorption and metabolism. For this test $1.0 \mathrm{gm}$ dextrose $/ \mathrm{kg}$ BW is delivered as a $50 \%$ solution by nasogastric tube after a 12 hour fast. Venous blood samples are taken at $30 \mathrm{~min}$ intervals for over 3 hours (Jacobs and Bolton 1982). This test is not practical for use in the field and it causes non-physiologic elevations in blood glucose and insulin that may obscure more subtle alterations in glucose/insulin metabolism (Ralston 2004).

The low dose oral dextrose challenge (LDOD) requires that only 0.20-0.25 gm dextrose/ $\mathrm{kg} \mathrm{BW}$ be given as a slurry with unsweetened applesauce or similar carrier by oral drench (Bergman et al. 1979, Hoffman et al. 2003b). This provides a glucose load roughly equivalent to a meal of grain. Even non-fasted nursing foals and weanlings have glucose and insulin responses at 60 and 90 min post dosing that are repeatable and within the physiologic limits normally seen after a meal of grain (Bergman et al. 1979). This allows a fairly non-traumatic, rapid assessment of glucose/insulin metabolism in a more controlled manner than the grain test. The test should be conducted in the morning between 07.00 and $10.00 \mathrm{hr}$. Blood samples should be drawn before dosing and 60-90 min later, the time at which peak responses are expected. Since gastric emptying and rate of ingestion are not as variable as with the grain meal, these sampling times can provide a fairly accurate assessment of the peak responses. Insulin responses are blunted after 10-12 months of age (Ralston 1996, Krusic et al. 1997, Ralston et al. 1998) and may not reflect abnormalities in glucose/insulin metabolism. Abnormal responses can be interpreted and acted upon as for the grain test. This should not be considered a definitive test, but rather a rapid screening test for gross abnormalities in glucose metabolism. More frequent sampling ( $q 15-30$ min) for a longer period of time (180 min) has been repeatable and reliable under a variety of experimental protocols (Bergman et al. 1979, Krusic et al. 1997, Ralston 2002, Ralston 2004).

\section{Factors affecting glucose/insulin metabolism in young horses}

There are many factors which must be taken into account when assessing glucose metabolism in any animal, such as age, previous diet, time of last meal or length of fast, etc. (Ralston 2002). Unfortunately there are many gaps in our understanding of what factors are of main importance in young horses. This section is a brief review of some of the data available.

\section{Ration}

Horses fed only forages have lower glucose tolerance (decreased rates of clearance) to standardized dextrose challenges than those fed rations containing 30-50\% high starch concentrates (Argenzio and Hintz 1972, Jacobs and Bolton 1982). Weanling fillies adapted to a high starch (45\%) concentrate fed at $50 \%$ of caloric intake, had lower glucose and insulin responses the LDOD than those fed a restricted starch (30\%) high fat (8\%) fiber (20\%) ration in equi-caloric amounts (Ralston 2003,2004 unpublished). Serum glucagon-like peptide 1 (GLP-1), a gastrointestinal hormone which stimulates pancreatic beta cell activity, was also increased in high starch fed fillies (Duarte and Ralston 2004 unpublished). As reported in other studies of high fat/fiber rations in horses (Jacobs and Bolton 1982, Hoffman et al. 2003), the high starch ration had a significantly higher glycemic index and insulin response than the high fat/fiber ration (Ralston 2003,2004 unpublished). Adult horses fed high starch versus high fat and fiber rations had more rapid clearance of a glucose load, higher insulin release but possibly reduced overall sensitivity to insulin when adapted to the high starch rations (Hoffman et al. 2003a, Hoffman et al. 2003b).

\section{Age}

In a study of Quarter Horse weanlings (Ropp et al. 2003) the glucose and insulin responses to a $2.2 \%$ fat (higher starch) ration were higher when the animals were 5 to 7 months old (day 0) than when they were 7 to 9 months of age (day 60). The authors of the report interpreted this as adaptation to the rations but it may instead reflect age-related changes in glucose tolerance. Standardbred 3 and Lippizaner 19 horses 3 12 months of age have been reported to have lower insulin sensitivity during standardized glucose challenges than when they were younger19 or 16-24 mo of age (Ralston 1996, Krusic et al. 1997). This coincides with period of highest growth hormone release and exogenously administered somatotropins reduce insulin sensitivity (Christensen et al. 1996), at least in adult horses. However insulin release in response to the LDOD in normal draft cross weanlings increased steadily with age from 6-18 months, regardless of rations being tested, with similar responses not being observed in adult horses on the same experiment (Ralston 2003,2004 unpublised). More work is needed on the effects of maturation on glucose metabolism in horses.

\section{Genetics}

Standardbred weanlings (4-7 mo of age) that had radiographic evidence of OCD lesions had higher insulin responses to a meal of concentrates than did radiographically normal weanlings that were the same age and fed the same rations (Ralston 1996, Ralston et al. 1998). The abnormal response was not apparent when the animals were re-tested at 18 months of age (Ralston 1996). Hereditary predisposition to at least hock OCD is well documented in several breeds (Rich and Brever 2002, Vervuert et al. 2003). The author's hypothesis is that a genetic defect that results in increased insulin resistance during the periods of rapid growth when the horses are most likely to be fed high glycemic index feeds (3-6 months) might be the proximal cause. Again, further in depth studies of genetically predisposed weanlings are needed.

\section{Exercise}

Exercise has been documented to improve insulin sensitivity in mature obese ponies (Freestone et al. 1992). Mild forced 
exercise (30 min walk/trot on a treadmill 3 times per week) also enhanced insulin sensitivity/glucose clearance in nonobese yearling Standardbred fillies (Black et al. 1997). Though not documented in young horses to date, obesity is associated with reduced glucose tolerance and hyperinsulinemia adult horses (Jeffcott et al. 1986), and is ameliorated by exercise.

\section{Plasma Cortisol (stress, time of day)}

Diurnal or stress induced elevations in plasma cortisol will also reduce glucose tolerance (Ralston 2002, Ralston 2004, Jeffcott et al. 1986). Glucose/insulin responses to a standardized challenge will be higher in unstressed horses in the morning than in the early afternoon but psychological stress will over ride the normal diurnal fluctuation (Ralston 2002).

\section{Conclusion}

The insulin-modified frequently sampled intravenous glucose tolerance test and low dose oral dextrose challenge offer a more easily administered and accurate assessment of glucose metabolism in young horses the experimental setting than some of the more traditional tests. A low dose oral dextrose challenge offers a practical field test for abnormal glucose/insulin responses. However, interpretation of the results of these tests must take into account the age of the horses tested, previous ration, time of day and degree of stress experienced during the test. In addition genetics and level of exercise/body condition may influence results. We have more questions than answers at this time regarding regulation of glucose/insulin metabolism in horses.

\section{References}

Argenzio R. A. and Hintz H. F. (1972): Effect of diet on glucose entry and oxidation rate in ponies. J Nutr 102, 879-903

Bergman R. N., Ider Y. Z., Bowden C. R. and Cobelli C. (1979): Quantitative estimation of insulin sensitivity. American J Physiology 236, E667-E677

Black A., Ralston S. L., Shapses S. A. and Schoknecht P. A. (1997): Skeletal development in weanling horses in response to high dietary energy and exercise. J Anim Sci. 75, 170

Cartmill J. A., Thompson L., Storer W. A., Gentry R. and Huff N. K. (2003): Endocrine responses in mares and geldings with high body condition scores grouped by high vs. low resting leptin concentrations. J Animal Science 81, 2311-2321

Christensen R. A., Malinowski K., Ralston S. L., Scanes C. G. and Hafs H. D. (2004): Chronic effects of equine growth hormone (eGH) on postprandial changes in plasma glucose, non-esterified fatty acids and urea nitrogen in aged mares. J Animal Science 74, 226

Duarte S. S. R. and Ralston S. L. (2004): Unpublished data

Freestone J. F., Beadle R., Shoemaker K., Bessin R. T., Wolfsheimer K. J. and Church C. (1992): Improved insulin sensitivity in hyperinsulinaemic ponies through physical conditioning and controlled feed intake. Equine Vet J 24,184-186

Garcia M. and Beech J. (1986): Equine intravenous glucose tolerance test: Glucose and insulin responses of healthy horses fed grain and hay and of horses with pituitary adenoma. Am. J. Vet Res $47,570-572$
Hoffman R. M., Boston R. C., Stefanovski D., Kronfeld D. S. and Harris P. A. (2003): Obesity and diet affect glucose dynamics and insulin sensitivity in Thoroughbred geldings. J. Animal Science 81, 2333-2342

Hoffman R. M., Kronfeld D. S., Cooper W. L. and Harris P. A. (2003a): Glucose clearance in grazing mares is affected by diet, pregnancy, and lactation. J. Animal Science 81, 1764-1771

Jacobs K. A. and Bolton J. R. (1982): The effect of diet on the oral glucose tolerance test in horses. J Am Vet Med Assoc 180, 884886

Jacobs K. A. and Bolton J.R. (1982): The effect of diet on the oral glucose tolerance test in horses. J. Am. Vet Med Assoc. 180, 884 886

Kronfeld D. S., Meacham T. N. and Donoghue S. (1990): Dietary aspects of developmental orthopedic disease in young horses. Veterinary Clinics of North America: Equine Practice. 6, 451-465

Jeffcott L. B., Field J. R., McClean J. G. and O'dea K. (1986): Glucose tolerance and insulin sensitivity in ponies and Standardbred horses. Equine Vet J 18, 97-101

Krusic L., Krusic-kaplia A., Cestnik V., Snoj T., Pogacnik A., Pangos S., Gatta D. and Moni P. (1997): Insulin response after oral glucose application in growing Lipizzaner foals. Proceedings of the 15th Equine Nutrition and Physiology Symposium, Fort Worth, TX, 15, 397-402

Pagan J. D., Geor R. J. and Caddell S. E. (2001): The relationship between glycemic response and the incidence of OCD in Thoroughbred weanlings. Proceedings of the 47th Annual American Association of Equine Practitioners Meetings, San Diego, CA, 48, 322-325

Powell D. M., Reedy S. E., Sessions S. R. and Fitzgerald B. P. (2002): Effect of short-term exercise training on insulin sensitivity in obese and lean mares. Equine Vet J Suppl. 34, 81-84

Ralston S. L. (1996): Hyperglycemia/hyperinsulinemia after feeding a meal of grain to young horses with osteochondritis dissecans (OCD) lesions. Pferdeheilkunde 12, 320-322

Ralston S. L., Black A., Suslak-Brown L. and Schoknecht P. A. (1998): Postprandial insuline resistance associated with osteochondrosis in weanling fillies. J Animal Science 76 (supplement 1) 176, 1998

Ropp J. K., Raub R. H. and Minton J. E. (2003): The effect of dietary energy source on serum concentration of insulin-like growth factor-l, growth hormone, insulin, glucose, and fat metabolites in weanling horses. J. Anim Sci. 81, 1581-1589

Ralston S. L. and Duarte S. S. R. (2003): Unpublished data

Ralston S. L. (2002): Insulin and glucose regulation. Veterinary Clinics of North America: Equine Prac. 18, 295-304

Ralston S. L. (2004): Glucose intolerance and hyperinsulinemia. In: Equine Internal Medicine, 2nd ed. Reed SM, Bayly WM, Sellon DC. Editors, Saunders, St. Louis. MO, 1600-1601

Ralston S. L., Hrabinski D. and Brady S. (2001): Glucose tolerance testing in foals. Proc. 17th Equine Nutrition and Physiology Symposium.Lexington, KY, 182

Ralston S. L. (2003, 2004): Unpublished data

Rich G. A. and Brever L. H. (2002): Recent developments in equine nutrition with farm and clinic implications. Proceedings of the 48th Annual American Association of Equine Practitioners Meetings, Orlando, FL, 48, 24-40

Vervuert I., Coenen M., Borchers A., Granel M., Winkelsett S., Christmann L., Distl O., Bruns E. and Hertsch B. (2003): Growth rates and the incidence of osteochondrotic lesions in Hanoverian warmblood foals. Proceedings of the 18th Equine Nutrition and Physiology Symposium. East Lansing, MI, 18, 113-114

\section{S. L. Ralston}

New Brunswick, NJ

Ralston@aesop.rutgers.edu 